\title{
Previsiones de la gestión de tesorería en la industria petrolera del estado Zulia
}

\author{
Forecasts of treasury management in the Zulia state oil industry
}

\begin{tabular}{l|r}
\cline { 2 - 2 } & Nelsy Caridad \\
\hline Artículo recibido en mayo 2020 & nelcaridad@hotmail.com \\
Arbitrado en junio 2020 & ORCID: 0000-0002-6434-7244 \\
Publicado en septiembre 2020 & Universidad del Zulia, Venezuela \\
\hline
\end{tabular}

RESUMEN | La metodología aplicada en la investigación es de tipo descriptiva, por cuanto se pretendió describir las previsiones de la gestión de tesorería en la industria petrolera del estado Zulia, bajo un diseño no experimental, de campo y transeccional. La población quedo conformada por el personal responsable en las áreas de tesorería, a quienes se le aplicó una encuesta a través de un cuestionario con 5 alternativas de respuestas, contentivo de 9 ítems. La validez se realizó mediante el juicio de expertos y la confiabilidad se determinó por medio del coeficiente Alpha Cronbach, cuyo valor fue de 0,80 . Con respecto a la técnica de análisis se recurrió al método estadístico, haciendo uso de las frecuencias relativas y la media aritmética. Se determinó un muy alto nivel, de la planificación financiera, previsión financiera y posición de liquidez como parte de la gestión de tesorería en la industria petrolera del estado Zulia.

Palabras clave: Gestión de tesorería, planificación financiera, posición de liquidez, previsión financiera, previsiones

Keywords:

The methodology applied in the research is descriptive, in that it was intended to describe the forecasts of treasury management in the oil industry of the Zulia state, under a non-experimental, field and transectional design. The population was made up of the personnel responsible in the treasury areas, to whom a survey was applied through a questionnaire with 5 response alternatives, containing 9 items. Validity was made by expert judgment and reliability was determined using the Alpha Cronbach coefficient, whose value was 0.80 . Regarding the analysis technique, the statistical method was used, using the relative frequencies and the arithmetic mean. A very high level of financial planning, financial forecast and liquidity position was determined as part of the treasury management in the oil industry of the Zulia state.

Treasury management, financial planning, liquidity position, financial forecast, forecasts 


\section{INTRODUCCIÓN}

Actualmente la industria petrolera en el ámbito mundial, presta sus servicios para garantizar un beneficio común y mejorar cada día más su calidad de servicio en el sector petrolero, haciéndose más atractivos y competitivos en el mercado, obteniendo un flujo de pago efectivo de sus clientes para poder brindar mayor satisfacción en la prestación de sus servicios o la actividad que este desempeñe para obtención de ingresos.

Es por ello que, la industria con la finalidad de subsistir y de mantenerse competitiva en un entorno cada vez más complejo y de múltiples cambios, se ven en la necesidad de enfocarse y centrar más su atención en una de las actividades principales, como lo es la tesorería, ya que dentro de ella está involucrado el manejo del activo liquidable a corto plazo (circulante), es decir el dinero disponible de las empresas, así como también indicadores importantes de gestión como las cobranzas, créditos, desembolsos, inversiones, valores realizables, transferencia de fondos, entre otros.

Todos estos elementos antes mencionados, permiten determinar el nivel de solvencia de la industria, tomando en cuenta que mientras más solvente se encuentre la misma, mayor capacidad tendrá para cubrir sus gastos operativos, incluso para cumplirle a sus proveedores, colocándose en una posición privilegiada dentro de su mercado, reduciendo al menos de forma considerable, toda situación de riesgo e inseguridad que pudiera presentarse, dentro de lo que tiene que ver con el ámbito financiero.

Sin embargo, el hecho de poseer un nivel elevado de solvencia, no garantizará que siempre se va a estar en presencia de un ambiente de total estabilidad, debido a que la industria petrolera no funciona de manera aislada, por lo que están sujetas a todo cambio - situación desfavorable que ocurra a su alrededor; es allí donde los gerentes deben efectuar una revisión de sus estrategias y replantear sus políticas para de alguna forma tratar de contrarrestar tal situación de incertidumbre. Desafortunadamente, muchos líderes no han sido certeros en cuanto a manejar esta clase de eventos, mostrando dificultades a la hora de tomar decisiones relevantes, perjudicando notablemente a la industria.

Así las cosas, una de las alternativas para enfrentar estas condiciones es manejar de manera efectiva y oportuna la gestión de tesorería, es decir, en correspondencia con lo que dice Van Horne (1999), donde la gestión del activo circulante de la organización, involucra el efectivo con que cuenta la empresa para operar, lo cual se hace posible estudiando la situación actual y determinando las características de la gestión en las actividades de cobranza, manejo de inventarios, control de desembolsos, inversiones en valores realizables y mecanismos de transferencia que permitan la administración del flujo de efectivo desplazando los fondos entre cuentas bancarias.

Ahora bien, según un diagnóstico realizado por la investigadora, a través de la revisión de datos en la página oficial de PDVSA (2016), la industria petrolera, específicamente en el estado Zulia, realizo apertura en procesos de licitación de ventas de activos de las bases cerradas para la recuperación de capital, así mismo, se llevó a cabo la posible fusión de grandes empresas dentro de la industria para nuevos proyectos de inversión, aumentando sus ingresos en la participación del mercado petrolero, sin embargo, el gobierno de Estados Unidos 
decidió declinar la fusión para no formar un monopolio.

Posteriormente, según cifras de la OPEP (2017), se registran declinaciones en las operaciones de los pozos activos por retraso en las cuentas por cobrar de las empresas mixtas que integran la industria petrolera del estado Zulia, debido en el cumplimiento de los acuerdos firmados de pago por parte del presidente de la industria petrolera con el presidente de las empresas mixtas y el cumplimiento de sus obligaciones.

De este modo, la industria petrolera situada en el estado Zulia, se encuentra en suspensión de sus operaciones, por la falta de cobro con las empresas mixtas de los acuerdos firmados, por cuanto por razones de respuesta, ha ocasionado incumplimiento de sus obligaciones, retraso en el pago de los trabajadores y proveedores, entre otras consecuencias que ocasiona la ineficiencia en la gestión de tesorería que ocasiona en la industria sea poca atractiva en el negocio de grandes reinversiones en la continuidad de las operaciones de la industria petrolera.

Bajo esta óptica, se presenta como trabajo de investigación describir las previsiones de la gestión de tesorería en la industria petrolera del estado Zulia, donde se demuestre la oportuna o no toma de decisiones de los diferentes departamentos que este involucra.

\section{Gestión de tesorería}

Según Gitman (2000), la gestión de tesorería, consiste en mantener niveles de saldo de efectivo para las transacciones que contribuyan a elevar el valor de la empresa. En este sentido, si los niveles de efectivo son demasiado altos, la rentabilidad de una empresa sería menor que si jugara con esos saldos para mantener saldos óptimos donde no existiera ni excedente ni déficit, es decir el estado ideal.

En el mismo orden de ideas, para Santandreu (2000), la gestión de tesorería es aquella área de gestión cuya misión radica en considerar esa actividad como un centro de movimientos monetarios, con independencia de la actividad propia económica de la empresa. Refiere el autor, que la tesorería por lo general no produce intereses, pero la gente prudente sigue manteniéndola, en lugar de invertirla en títulos que produzcan intereses, debido a que ésta proporciona más liquidez que los títulos.

En coincidencia con lo expuesto, González (2009), define la gestión de tesorería como la diferencia entre la parte del capital que tiene la empresa, no está dedicada a financiación del inmovilizado y que, a su vez está disponible para poder financiar el ciclo productivo, mientras que Olsina (2009) la denomina como cash management.

A efectos de esta investigación, la gestión de tesorería, consiste en la optimización de la liquidez, con el fin de garantizar la disponibilidad de los fondos necesarios para la realización de los pagos comprometidos por la industria petrolera del estado Zulia, en la divisa adecuada y en el momento preciso.

\section{Previsiones de la gestión de tesorería}

Olsina (2009) detalla las previsiones en la gestión de tesorería como las medidas que se deben tomar para determinar los posibles riesgos asociados ante un cambio de decisiones. Asimismo, Santandreu (2010) menciona, son escenarios que pueden llegarse a cumplirse sino se posee una vigilancia ante esos riesgos que deben reducirse al máximo para lograr sus objetivos en la industria. 
Por su parte, González (2009), la define como la mejor medida ante de tomar una decisión en una negociación y evaluar cambios en los supuestos que garanticen la rentabilidad de la industria ante cualquier supuesto, podrían colocar sus indicadores financieros en posible zona de riesgo, de igual modo se debe constituir una seria de interrogantes que darán respuestas certeras en los cambios.

A criterio de la investigadora, las previsiones de la gestión de tesorería, son los movimientos futuros, reales o previstos, que permiten anticiparse e imaginar la situación en que se puede estar en un momento dado, esto se puede lograr a través de la planificación financiera, previsión financiera y posición de liquidez.

\section{Planificación financiera}

Hacer referencia a la planificación financiera, es visualizar una herramienta de apoyo a la gestión de tesorería de cualquier empresa, en función de los múltiples beneficios que puede aportar al eficaz desenvolvimiento de los procesos operativos contables. En este sentido, es abordado el tema, a partir de la visión de teóricos estudiosos de área:

Se inicia este recorrido examinando los aportes de Cibrán (2013), quien considera a la planificación financiera como aquellos datos que, al ser cuantificados en los planes de una organización, generan una ocurrencia directa en su patrimonio económico. De allí, que enfatiza en el hecho que el inicio del diagnóstico se hace partiendo de las insuficiencias que en materia financiera padece la estructura organizacional analizada, tanto en su valor como en las condiciones que las originan. En consecuencia, es requerido realizar una valoración, a los fines de medir la importancia del déficit existente, para comprobar las razones o síntomas que permitan determinar la causa del problema.

En síntesis, parafraseando al citado autor, la planificación financiera puede conceptualizarse como aquella información contable, que es cuantificada de los planes de la empresa y además por su naturaleza incide sobre los recursos financieros de la misma. De igual forma, puede ser considerada como proceso, por la secuencia de pasos a seguir para la obtención de un plan. Asimismo, es necesario tener en cuenta que la calidad de sus resultados, es garantizada por la veracidad de la información contable esgrimida para su preparación.

De acuerdo a la opinión esbozada por Ortega (2008), en la planificación financiera hace falta la consolidación de la planeación, así como el presupuesto. Porque planificar implica proyectar hacia un futuro la proyección del negocio. Por lo tanto, al momento de visualizar de manera prospectiva esta fase del proceso organizativo, se está minimizando la posibilidad de riesgos a los que puede enfrentarse la empresa en un determinado momento del tiempo.

Continuando con la revisión teórica, corresponde examinar lo expuesto por Morales y Morales (2014), autores impulsadores de la idea que la planeación financiera implanta la manera de alcanzar las metas establecidas $u$ objetivos propuestos, debido a que facilitan acciones que tutelan, sistematizan y vigilan las actividades necesarias para el cumplimiento de esos objetivos, en consecuencia, el plan financiero, según los autores, tiene que emerger de un plan general establecido por la dirección de la organización, esa alineación entre ambos es indispensable para garantizar el éxito, sin la presencia de esta variable, es posible una 
desviación en el camino a recorrer para lograr esa tan ansiada meta, significando este acontecimiento una posibilidad cierta de distracción de recursos en movimientos que no benefician a la empresa, sino por el contrario absorben tiempo y capitales.

En correspondencia con lo planteado anteriormente, se aborda la posición de Paredes (2017), para quien, la planificación financiera es considerada como un proceso que forma parte de los mecanismos clave para la efectiva y eficaz misión empresarial, por ello, la puesta en práctica de este proceso incrementa la posibilidad de que la organización alcance los objetivos formulados en su plataforma filosófica, a través de un plan estratégico.

Desde estas perspectivas, para la investigadora, la planificación financiera, es un enfoque objetivo y sistemático que permite a la empresa asumir una posición proactiva y no reactiva en el mercado que compite, ya que es mejor adelantarse a los acontecimientos que estar a la espera de lo que pueda suceder.

\section{Previsión financiera}

González (2009) establece, la falta de liquidez de la empresa forma parte focal para tomar previsiones en minimizar el riesgo en el funcionamiento diario a la actividad, la misma forma parte elemental de gestión que sirve para interferir la liquidez en cada momento, en función de recoger detalladamente los cobros y pagos que realiza de mes a mes en el negocio, por tanto, se debe distinguir claramente los ingresos de los cobros, posteriormente los gastos de los pagos.

Asimismo, Hernández (2014) define la previsión como antecedente inmediato para una buena administración. Prever significa calcular el porvenir y prepararlo, de programar la acción por ejecución del plan para poder actuar en el mismo, su principal manifestación, su signo sensible, su instrumento más eficaz, es el programa de acción, es a la vez el resultado a que se tiende, la línea de conducta que se ha de seguir.

Basado en estos enfoques, para la investigadora, la previsión financiera, es aquella que ofrece la visión a largo plazo sobre las inversiones de una empresa, a la vez que ayuda a analizar los momentos más propicios para llevarla a cabo.

\section{Posición de liquidez}

González (2009) establece dos grandes problemas en la gestión de tesorería: falta de liquidez o exceso de liquidez, la primera se refiere a la falta de dinero líquido para cumplir con sus obligaciones, algunos de los motivos que existen para la diminución de liquidez se pueden conseguir los siguientes factores que a muchas empresas a veces les genera beneficios que pueden ser de diversa índole:

$\checkmark$ Financiación de activos fijos mediante la tesorería de la empresa o al corriente: se incurre cuando se adquiere una máquina y se paga al contado con dinero que se tenga en caja o bancos, allí se pierde liquidez para afrontar con los pagos corrientes de la empresa.

$\checkmark$ No tener en cuenta el periodo de flujo de caja: es el tiempo que transcurre desde hago una inversión hasta que la recupero mediante la venta de las mercancías o la prestación de servicios. Cuando esto sucede, la solución está en intentar acortar el plazo de cobro y alargar el de pago. De no conseguirlo habrá que recurrir a la financiación externa. 
$\checkmark$ Mala gestión del IVA. La gran problemática del IVA es que el devengo del impuesto puede producirse antes de que la empresa ingrese ese IVA, por ejemplo, cuando se tienen períodos de cobro muy largos. Esto puede dar lugar a que se tenga que ingresar un IVA que aún no se cobró, lo que puede disminuir mucho la liquidez. Esto, de hecho, es uno de los problemas que más afectan a las Pymes y Autónomos.

$\checkmark$ Falta de control y gestión en los stocks, particularmente de existencias.

Algunas empresas suelen hacer aprovisionamientos mediante grandes compras, por cuanto, de esta forma, pueden acceder a un mejor precio de adquisición. Si no se gestionan bien los stocks, se desembolsarán grandes cantidades por las compras disminuyendo así la tesorería de la empresa. Hay que tener en cuenta que una buena política de compras no sólo es comprar barato, sino calcular la cantidad idónea que se va a vender en el plazo de reposición de la compra.

Algunas herramientas para evitar la falta de liquidez son las siguientes: crédito comercial, descuentos de efectos comerciales liberados a los clientes, créditos y préstamos bancarios a corto plazo, por último, tratar de anticipar los cobros a los clientes. De esta manera se pueden establecer estas series de herramientas para luchar contra una situación de falta de liquidez para que las empresas cumplan con sus obligaciones.

La segunda no se puede considerar como un problema propiamente dicho, el exceso de liquidez tiene un coste de oportunidad para el autónomo. Si este exceso de liquidez se mantiene en caja no generaría rendimiento y si se mantiene en las cuentas bancarias generaría un rendimiento muy pequeño. Debido a esto hay que buscar usos alternativos para utilizar este exceso. En este sentido, los usos más interesantes para el autónomo son:

$\checkmark$ Anticipar el pago a los proveedores para conseguir descuentos por pronto pago.

$\checkmark$ Amortizar anticipadamente créditos o préstamos en vigor. Se disminuirán así los costes derivados de los intereses del crédito

$\checkmark$ Conceder mayor plazo de pago a clientes. Gracias al exceso de liquidez podemos permitir que los clientes que tenemos tarden más en pagarnos, con que facilitaremos las ventas.

$\checkmark$ Invertir el exceso de tesorería en activos financieros que ofrezcan mayor rentabilidad, asegurando la liquidez. Se obtendrá así un mayor rendimiento que el que se obtiene de los intereses de una cuenta corriente.

Mientras que para Santandreu (2010), los excedentes deben permanecer en la empresa cierto tiempo, pero no se desea prescindir del mismo, existen también otras formas de aplicar el ahorro, aunque, como se ha dicho a largo plazo. Algunas de dichas formas son: fondos de inversión. Se trata de patrimonios creados por muchos inversores que constituyen un fondo común denominado fondo de inversión. Los fondos de inversión actualmente suponen unas alternativas interesantes, aunque según las características de cada uno de ellos, ofrecen menor rentabilidad por estar invertidos en títulos de renta fija, o mayor rentabilidad si lo están en renta variable. Planes y fondos de ahorro y previsión.

Para Olsina (2009), la posición de liquidez en que se encuentre la empresa menciona tener en cuenta los saldos de lo que conforma la 
estructura financiera que deben estar forjadas en la interpretación y el manejo del tesorero que ayude a la toma de decisiones, en conducir a la empresa al logro de sus objetivos, debe estar evaluado en la previsión del tesorero para disminuir ambas situaciones tanto la falta o excedente de liquidez.

Para la investigadora, la posición de liquidez, es la capacidad que posee una empresa para pagar sus obligaciones a corto plazo en la medida que se vencen. De manera que, se considera que la liquidez está referida a la solvencia de la posición financiera general de la organización, lo que se traduce en la facilidad que tiene la empresa para pagar sus deudas.

\section{MÉTODO}

La metodología aplicada en la presente investigación es de tipo descriptiva, bajo un diseño no experimental, de campo y transeccional. La población quedo conformada por los gerentes financieros, analistas de finanzas, analistas de tesorería y tesoreros adscritos a los departamentos de finanzas y tesorería de la industria petrolera venezolana, en el estado Zulia, para un total de 12 sujetos informantes.

Se aplicó una encuesta a través de un cuestionario, contentivo de 9 ítems, con 5 alternativas de respuestas: siempre (5); casi siempre (4); a veces (3); casi nunca (2) y nunca (1). La validez se realizó mediante el juicio de expertos y la confiabilidad se determinó por medio del coeficiente Alpha Cronbach, cuyo valor fue de 0,80 . Con respecto a la técnica de análisis se recurrió al método estadístico, haciendo uso de las frecuencias relativas y la media aritmética, utilizando los baremos establecidos para la interpretación de ambos estadísticos, mostrados en el cuadro 1 y 2.

Cuadro 1. Baremo para la interpretación de la media aritmética

\begin{tabular}{ccc}
\hline INTERVALO & CATEGORÍA & INTERPRETACIÓN \\
\hline $4.21-5.00$ & Siempre & Muy alto nivel \\
$3.41-4.20$ & Casi siempre & Alto nivel \\
$2.61-3.40$ & A veces & Moderado nivel \\
$1.81-2.60$ & Casi nunca & Bajo nivel \\
$1.00-1.80$ & Nunca & Muy bajo nivel \\
\hline
\end{tabular}

Fuente: Elaboración propia (2019)

Cuadro 2. Categoría de análisis para la frecuencia relativa

\begin{tabular}{cc}
\hline Alternativa & Tendencia \\
\hline $\mathrm{S}+\mathrm{CS} \geq 75 \%$ & La tendencia se considera positiva \\
$45 \% \leq \mathrm{S}+\mathrm{CS}<75 \%$ & La tendencia se considera neutral \\
$\mathrm{S}+\mathrm{CS}<45 \%$ & La tendencia se considera negativa \\
\hline
\end{tabular}

Fuente: Elaboración propia (2019) 


\section{RESULTADOS}

Como puede observarse en la tabla 1 , un $88,89 \%$ en promedio de los encuestados respondieron a los ítems involucrados en las alternativas siempre y casi siempre, indicando tendencia positiva. Asimismo, se observa para cada uno de los ítems aplicados una concentración de respuestas en estas opciones, cuyos valores ascienden a 83,33\%, 83,33\%, y 100\% respectivamente, marcando tendencias positivas.

Tabla 1. Indicador: Planificación financiera

\begin{tabular}{l} 
Ítems \\
\hline 1. En la industria petrolera se realizan las relaciones de gastos y ganancias, para el \\
presupuesto anual. \\
2. En la industria petrolera existen planes de expansión en la prestación de servicios. \\
3. En la industria petrolera se realiza la planificación de equipos en la prestación de servicio \\
para un periodo de actividad de un año.
\end{tabular}

\begin{tabular}{|c|c|c|c|c|}
\hline \multirow{3}{*}{ Categoría } & \multicolumn{4}{|c|}{ Ítems/Frecuencias } \\
\hline & 1 & 2 & \multicolumn{2}{|c|}{3} \\
\hline & FA & FA & FA & FR \\
\hline Siempre & 58,33 & 50,00 & 10 & 83,33 \\
\hline Casi siempre & 25,00 & 33,33 & 2 & 16,67 \\
\hline A veces & 16,67 & 16,67 & 0 & 0 \\
\hline Casi nunca & 0 & 0 & 0 & 0 \\
\hline Nunca & 0 & 0 & 0 & 0 \\
\hline Total & 100 & 100 & 12 & 100 \\
\hline Alternativa Ítems & $\begin{array}{c}83,33 \% \\
S+C S \geq 75 \%\end{array}$ & $\begin{array}{c}83,33 \% \\
S+C S \geq 75 \%\end{array}$ & $S+C S \geq 75 \%$ & \\
\hline Tendencia Ítems & Positiva & Positiva & \multicolumn{2}{|c|}{ Positiva } \\
\hline Alternativa Indicador & \multicolumn{2}{|r|}{$S+C S \geq 75 \%$} & $88,89 \%$ & \\
\hline Tendencia Indicador & \multicolumn{4}{|c|}{ Positiva } \\
\hline \multirow{3}{*}{$\begin{array}{l}\text { Promedio de las respuestas } \\
\text { codificadas }\end{array}$} & \multicolumn{4}{|c|}{ Ítems } \\
\hline & 1 & 2 & \multicolumn{2}{|c|}{3} \\
\hline & 4,42 & 4,33 & \multicolumn{2}{|c|}{4,83} \\
\hline Categoría & Muy alto nivel & Muy alto nivel & \multicolumn{2}{|c|}{ Muy alto nivel } \\
\hline \multirow[t]{2}{*}{ Promedio Indicador } & & 4,53 & & \\
\hline & & Muy alto nivel & & \\
\hline
\end{tabular}

Fuente: Elaboración propia (2019) 
Al mismo tiempo se muestra una media de 4,52 para este indicador, implicando muy alto nivel de la planificación financiera como una herramienta de apoyo a la gestión de tesorería de la industria petrolera del estado Zulia. Dicha media está determinada por el comportamiento de las medias a las que arribaron cada uno de los ítems aplicados. De esta manera se observa, para el ítem 1 una media de 4,42, indicando muy alto nivel de las relaciones de gastos y ganancias, para el presupuesto anual que ejecuta la industria petrolera.

Adicionalmente, se evidencia muy alto nivel el que: en la industria petrolera existen planes de expansión en la prestación de servicios $(4,33)$; y se realiza la planificación de equipos en la prestación de servicio para un periodo de actividad de un año $(4,83)$.

Los resultados alcanzados, a juicio de la investigadora, permiten afirmar que la industria petrolera en estudio, cuenta con un proceso de elaboración de un plan financiero integral, organizado, detallado y personalizado, que garantiza alcanzar los objetivos financieros determinados previamente, así como los plazos, costes y recursos necesarios para que sea posible.

Esta situación mostrada, pone en evidencia muy alta coincidencia con Morales y Morales (2014), para quienes, la planeación financiera implanta la manera de alcanzar las metas establecidas u objetivos propuestos, debido a que facilitan acciones que tutelan, sistematizan y vigilan las actividades necesarias para el cumplimiento de esos objetivos, en consecuencia, el plan financiero, según los autores, tiene que emerger de un plan general establecido por la Dirección de la Organización.

Asimismo, validan a Paredes (2017), para quien, la planificación financiera es considerada como un proceso que forma parte de los mecanismos clave para la efectiva y eficaz misión empresarial, por ello, la puesta en práctica de este proceso incrementa la posibilidad de que la organización alcance los objetivos formulados en su plataforma filosófica, a través de un plan estratégico.

Con base a lo descrito, puede decirse entonces que, en la industria petrolera estudiada, se maneja la planificación financiera con muy alto criterio como indicador de las previsiones de la gestión de tesorería, validando con ello, la posición de la investigadora, quien la visualiza como un enfoque objetivo $y$ sistemático que permite asumir una posición proactiva y no reactiva en el mercado que compite.

En lo concerniente al indicador previsión financiera, se observa, en la tabla 2, un promedio de frecuencias de $91,67 \%$ en las opciones positivas (siempre y casi siempre), indicando tendencia positiva de las respuestas emitidas. En este aspecto, al detalle se observa que la suma de las opciones para cada uno de los ítems se posiciona en la tendencia positiva. 
Tabla 2. Indicador: Previsión financiera

\section{Ítems}
4.
En la industria petrolera se realiza plan de previsión a través de presentaciones de la situación financiera.
5.
En la industria petrolera se optimizan los recursos de trabajo a través de las ventas de equipos o activos depreciados para aumentar sus ingresos.
6.
En la industria petrolera se realizan proyecciones semestrales para poder minimizar el riesgo financiero.

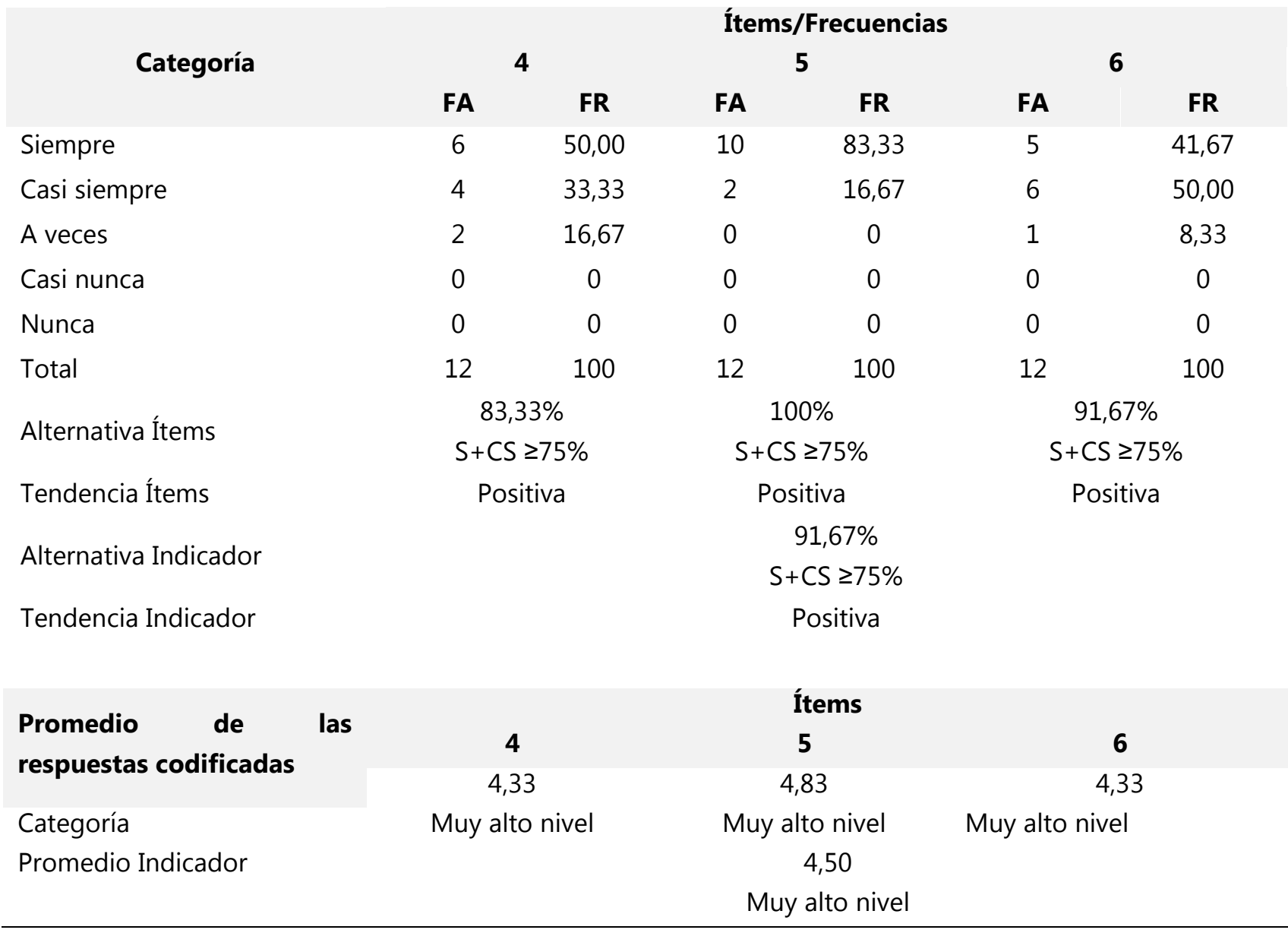

Fuente: Elaboración propia (2019)

Adicional a ello, se evidencia un promedio de 4,50 ubicándolo en la categoría de muy alto nivel de la previsión financiera como los resultados de una planificación en la gestión de tesorería de la industria petrolera del estado Zulia. Al detalle se observa muy alto nivel para los ítems 4,5 y 6 indicando que se cataloga con muy alto nivel que: en la industria petrolera se realiza plan de previsión a través de presentaciones de la situación financiera $(4,33)$; se optimizan los recursos de trabajo a través de las ventas de equipos o activos depreciados para aumentar sus ingresos $(4,83)$ y se realizan proyecciones semestrales para poder minimizar el riesgo financiero $(4,33)$. 
Según los resultados mostrados, la investigadora considera que la previsión financiera sugiere la estimación de un resultado futuro a partir de la planificación del negocio de las operaciones, y su efectiva utilidad radica en la evaluación el impacto de los objetivos y decisiones de gestión en la situación financiera general de la industria.

Lo mostrado alcanza a validar, de manera muy alta, lo postulado por Hernández (2014), quien define la previsión como antecedente inmediato para una buena administración. Prever significa calcular el porvenir y prepararlo, de programar la acción por ejecución del plan para poder actuar en el mismo, su principal manifestación, su signo sensible, su instrumento más eficaz, es el programa de acción, es a la vez el resultado a que se tiende, la línea de conducta que se ha de seguir.

Al mismo tiempo, se corresponde con la opinión de la investigadora, quien considera que la previsión financiera es aquella que ofrece la visión a largo plazo sobre las inversiones de una empresa, a la vez que ayuda a analizar los momentos más propicios para llevarla a cabo.

En la tabla 3 se resume el comportamiento del indicador posición de liquidez, donde se observa que el mismo alcanzó un promedio de frecuencias de $86,11 \%$ en las opciones siempre y casi siempre, indicando tendencia positiva de las respuestas. De manera específica, puede observarse para cada uno de los ítems aplicados una concentración de respuestas en las opciones siempre y casi siempre, cuyos valores ascienden a 7, 8 y 9 el 100\%, 83,33\% y 75\% respectivamente, marcando tendencias positivas.

Adicionalmente, la tabla muestra un promedio de 4,39 lo cual según el baremo establecido ubica al indicador en la categoría de muy alto nivel. Esto se refleja en sus medias de 4,$67 ; 4,33$ y 4,17 respectivamente, indicando según opinión de los encuestados, muy alto nivel de la posición de liquidez como la capacidad para obtener dinero en efectivo y así hacer frente a sus obligaciones a corto plazo, fortaleciendo la gestión de tesorería de la industria petrolera del estado Zulia.

Al desglosarse los resultados, se evidencia con muy alto nivel que en la industria petrolera se realiza monitoreo de situación financiera $(4,67)$ y posee instrumento de medición de liquidez $(4,33)$; no obstante, con alto nivel se realizan tablas en los que se muestra la situación financiera $(4,17)$. 
Tabla 3. Indicador: Posición de liquidez

\section{Ítems}

7. En la industria petrolera se realiza monitoreo de situación financiera.

8. La industria petrolera posee instrumento de medición de liquidez.

9. En la industria petrolera se realiza tablas en los que se muestra la situación financiera.

\begin{tabular}{|c|c|c|c|c|}
\hline \multirow{3}{*}{ Categoría } & \multicolumn{4}{|c|}{ Ítems/Frecuencias } \\
\hline & 7 & 8 & \multicolumn{2}{|c|}{9} \\
\hline & FA & FA & FA & FR \\
\hline Siempre & 66,67 & 50,00 & 5 & 41,67 \\
\hline Casi siempre & 33,33 & 33,33 & 4 & 33,33 \\
\hline$A$ veces & 0 & 16,67 & 3 & 25,00 \\
\hline Casi nunca & 0 & 0 & 0 & 0 \\
\hline Nunca & 0 & 0 & 0 & 0 \\
\hline Total & 100 & 100 & 12 & 100 \\
\hline Alternativa Ítems & $100 \%$ & $83,33 \%$ & \multicolumn{2}{|c|}{$75 \%$} \\
\hline Tendencia Ítems & Positiva & Positiva & \multicolumn{2}{|c|}{ Positiva } \\
\hline Alternativa Indicador & \multicolumn{4}{|c|}{$86,11 \%$} \\
\hline Tendencia Indicador & \multicolumn{4}{|c|}{ Positiva } \\
\hline \multirow{3}{*}{$\begin{array}{l}\text { Promedio de las respuestas } \\
\text { codificadas }\end{array}$} & \multicolumn{4}{|c|}{ Ítems } \\
\hline & 7 & 8 & & 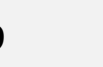 \\
\hline & 4,67 & 4,33 & & 17 \\
\hline Categoría & Muy alto nivel & Muy alto nivel & & nivel \\
\hline \multirow[t]{2}{*}{ Promedio Indicador } & \multicolumn{4}{|c|}{4,39} \\
\hline & & Muy alto nivel & & \\
\hline
\end{tabular}

Fuente: Elaboración propia (2019)

Así, para la investigadora, los resultados demuestran que, según los encuestados, la industria petrolera mantiene un muy alto nivel de liquidez, lo que es fundamental para su supervivencia ya que supone la capacidad que tiene para hacer frente a sus obligaciones de pago.

Lo mostrado valida, en muy alto grado, lo postulado por Olsina (2009), para quien la posición de liquidez en que se encuentre la empresa menciona tener en cuenta los saldos de lo que conforma la estructura financiera que deben estar forjadas en la interpretación y el manejo del tesorero que ayude a la toma de decisiones, en conducir a la empresa al logro de sus objetivos, debe estar evaluado en la previsión del tesorero para disminuir ambas situaciones tanto la falta o excedente de liquidez.

De igual manera, se valida lo expresado por la investigadora, cuando afirma que la posición de liquidez, es la capacidad que posee una 
empresa para pagar sus obligaciones a corto plazo en la medida que se vencen. De manera que, se considera que la liquidez está referida a la solvencia de la posición financiera general de la organización, lo que se traduce en la facilidad que tiene la empresa para pagar sus deudas.

Ya analizados cada uno de los indicadores considerados en la dimensión denominada previsiones de la gestión de tesorería, se pasa a verificar los resultados arrojados para esta dimensión. En la tabla 4 se observa un promedio de respuestas de $88,89 \%$ ubicándose en el rango $S+C S \geq 75 \%$ con una interpretación de tendencia positiva; acompañado de una media de 4,47 indicando muy alto nivel, según el baremo diseñado. Los resultados observados demuestran un muy alto nivel, de la planificación financiera, previsión financiera y posición de liquidez como parte de la gestión de tesorería en la industria petrolera del estado Zulia.

Lo precedente valida, en muy alto grado, lo manejado por González (2009), para quien las previsiones son la mejor medida de la gestión de tesorería ante de tomar una decisión en una negociación y evaluar cambios en los supuestos que garanticen la rentabilidad de la industria ante cualquier supuesto.

Tabla 4. Dimensión: Previsiones de la gestión de tesorería

\begin{tabular}{|c|c|c|c|c|}
\hline Indicador & Alternativa & Categoría & Media & Categoría \\
\hline Planificación financiera & $\begin{array}{c}88,89 \% \\
S+C S \geq 75 \%\end{array}$ & Positiva & 4,53 & Muy alto nivel \\
\hline Previsión financiera & $\begin{array}{c}91,67 \% \\
S+C S \geq 75 \%\end{array}$ & Positiva & 4,50 & Muy alto nivel \\
\hline Posición de liquidez & $\begin{array}{c}86,11 \% \\
S+C S \geq 75 \%\end{array}$ & Positiva & 4,39 & Muy alto nivel \\
\hline Promedio & $\begin{array}{c}88,89 \% \\
S+C S \geq 75 \%\end{array}$ & Positiva & 4,47 & Muy alto nivel \\
\hline
\end{tabular}

Fuente: Elaboración propia (2019)

De igual manera, se valida lo expresado por la investigadora, para quien las previsiones de la gestión de tesorería, representan los movimientos futuros, reales o previstos, que permiten anticiparse e imaginar la situación en que se puede estar en un momento dado, esto se puede lograr a través de la planificación financiera, previsión financiera y posición de liquidez.

Con base a lo mostrado para esta dimensión, se deduce que en la industria petrolera bajo estudio se tiene muy alta consideración de la planificación financiera, previsión financiera y posición de liquidez como parte de la gestión de tesorería, a través de la cual se busca la optimización de la liquidez, con el fin de garantizar la disponibilidad de los fondos necesarios para la realización de los pagos comprometidos por la industria petrolera del estado Zulia, en la divisa adecuada y en el momento preciso. 
CONCLUSIONES

Tomando en cuenta los resultados del indicador se puede decir que la industria petrolera del estado Zulia utiliza las relaciones de gasto para el presupuesto anual, existen planes de expansión en la prestación de servicio y la planificación de equipos para la actividad de un año, garantizando sus operaciones a la medida de las exigencias del cliente. Así las cosas, las previsiones de la gestión de tesorería son eficientes herramientas para analizar la capacidad de la industria para determinar las necesidades de efectivo, midiendo su desempeño y eficiencia en la administración de los activos, pasivo y el capital.

No obstante, de cara a la realidad palpable en este análisis, las evidencias de la dificultad financiera que exhibe actualmente el país, son tales que las conclusiones son más que obvias. En tal sentido, se requiere una efectiva gestión de tesorería, a fin de mantener niveles de saldo de efectivo para las transacciones que contribuyan a elevar el valor de la industria petrolera.

\section{REFRENCIAS}

Cibrán, P (2013). Planificación financiera. ESIC Editorial. España

Gitman, L. (2000). Principios de Administración Financiera. 8va Edición, Editorial Prentice Hill. México
González, A. (2009). Gestión de tesorería., C.E.E.I. Editorial, Galicia, S.A. Santiago de Compostela

Hernández, Z. (2014). Administración estratégica. Primera edición, Ebook. Grupo de editores patria. México

Morales, A. y Morales J. (2014). Planeación Financiera. Grupo Editorial Patria. México

Olsina, X. (2009). Gestión de tesorería (optimizando los flujos monetarios). Profit. Editorial. Barcelona

OPEP (2017). Monthly Oil Market Report (OPEC, IEA, OECD, WB Joint Report). Disponible en: http://www.opec.org/opec_web/en/publicat ions/338.htm.

Ortega, A. (2008). Planeación financiera estratégica. Mc Graw Hill. México

Paredes, J. (2017). Planificación financiera ante la perspectiva organizacional en empresas cementeras del estado Zulia-Venezuela. Económicas CUC, 38(1), 105-132.

PDVSA página oficial de internet (2016). Disponible en: http://www.pdvsa.com/index.php?tpl=interf ace.sp/design/readmenuprinc_eps.tpl.html \&newsid_temas $=220$

Santandreu, E. (2000). Manual de Finanzas. Ediciones Gestión 2000, S.A

Van Horne J. (1999). Administración Financiera. 10ma Edición. Editorial Prentice Hall Hispanoamericana, S.A. México 